Aus der chirurgischen Privatklinik von Prof. Dr. C. S. Haegler in Basel.

\title{
Die Fraktar des Condylus externus tibiae.
}

\author{
Von Dr. Hans Meerwein, Basel.
}

(Mit 5 Abbildungen.)

Die Frakturen am oberen Tibiaende gehüren bekauntlich zu den Seltenheiten. Indem wir die traumatischen Epiphysenłösungen, die Frakturen der 'Tuberositas tibiae, die neuerdings Jak 0 bsdahl'1) nnd Schlatter ${ }^{2}$ ) wieder in den Vordergrund des Interesses gerückt haben, sowie die Konpressionsfrakturen am oberen Tibiaende, die von Wagner ${ }^{3}$ ) eingehend beschrieben wurden, übergehen, sollen hier nur die isolierten Frakturen eines Tibiacondyls eine ausführlichere Besprechung erfahren. Auch sie gehören zu den selten vorkommenden Frakturen, sind aber wohl häufiger, als bisher angenommen wurde. Sonntag erwähnt, daß Heydenreich 1877 aus der gesamten Literatur nur 4 Fälle zusammenbringen und auch Démoulin 1994 kein weiteres Material beibringen konnte. Lö $\left.{ }^{4}\right)$ fand unter 2600 am Bürgerhospital zu Cöln (189ij-1897) beobachteten Frakturen 2 Fälle von Fraktur des Condylns internus tibiae. Beide waren durch direkte Gewalt entstanden. Sonntag selbst stellte 1906 die in den vorhergehenden 10 Jahren an der Tübinger Klinik beobachteten Frakturen am oberen Tibiaende zlsammen und fand unter diesem Material nur einen Fall einer Fraktur des Condylus externus bei einem Mann, der $6-7$ m hoch herab auf die Fïße gefallen war.

Die Fntstehung und die Symptome der Fraktur eines 'libiacondyls sind zwar typisch, die letzteren jedoch nicht so alarmierend, daß man sich unbedingt veranlaßt fühlte, eine Röntgenphotographie anfertigen zu lassen. Die Patienten künnen in der Regel noch

1) Jakobsdah!. Dentsche Zeitschr. f'. Chir., Bd. 86, S. 493.

2) Schlatter. Bruns' Beitr. Bd, 59, H. 3, S. 518 .

3) Wagner. Arch. f. klin. Chir. 1887, Bd. 34.

4) Deutsche Zeitschr. f. Chir., Bd. 44, S. 422 . 
gehen; etwas Schwellung im Gelenk ist nachweisbar, doch ist die aktive und passive Beweglichkeit im Kniegelenk nicht so erheblich eingeschränkt, wie man bei einem Knochenbruch erwarten dürfte. Diesem Umstand ist es zuzuschreiben, daßs wohl zahlreiche solcher Frakturen übersehen werden, indem man sich mit der Diagnose der Distorsion oder der Zerrung bzw. Zerreißung eines Seitenbandes begnügt; and doch treten bei Verkennung dieser Frakturen irreparable Störungen im Gelenkmechanismus auf, die dem Patienten zu dauerndem Schaden gereichen.

Wir hatten Gelegenheit, in den letaten 6. Monaten 4 solcher Fälle von Fraktur des äußeren Tibiacondyls zu beobachten; 3 davon waren von den behandelnden Ärzten nicht erkannt worden und kamen wegen der üblen Folgeerscheinungen in die Klinik; beim 4, der frisch eingeliefert wurde, machte anch uns die Stellung der Diagnose bis zur Anfertigung eines Röntgenogrammes Schwierigkeiten.

I. C. R., 30 jähriger Maurer, fiel am 9. V. 1908 infolge Umkippens einer Pflasterkiste, auf der er stand, ca. $3 \mathrm{~m}$ hoch herunter. Er kam aufrecht, mit den FüBen zuerst, auf den Dachboden und knickte dann mit den Knien nach vorn ein. Er richtete sich mit der Hand an einem Sparren auf; wegen starker Schmerzen im linken Knie mußte er die Arbeit aussetzen. Zuerst ging er mühsam an einem Stock, dann mit Unterstützung anderer Arbeiter nach Hause. Der zugezogene Arzt konstatierte eine Schwellung an der AuBenseite des linken Knies; ein GelenkerguB war angeblich weder damals noch später nachweisbar. Die passiven Bewegungen im linken Kniegelenk zeigten keine Einschränkung, waren aber sehr schmerzhaft; aktive Bewegungen wurden nicht ausgeführt. Die Gegend des Condylus externus tibiae war stark druckempfindlich. Der behandelnde Arzt stellte die Diagnose auf "Quetschung des linken Kniegelenks, besonders der AuBenseite des Gelenkanteils der Tibia " und verordnete Bettruhe und Umsehläge mit Bleiwasser. Schon nach 4 Tagen (1) konnte Patient wieder leidlich am Stock gehen und wurde von nun an täglich massiert; außerdem wurde passive und aktive Gymnastik getrieben. Ein nach 6 Wochen zugezogener zweiter Arzt stellte die Diagnose auf Zerreißung des inneren Seitenbandes, ev. mit Meniscusverletzung; Massage und Gymnastik wurden fortgesetzt; eine Röntgenaufnahme wurde nicht gemacht. Ende Juli konnte Patient wieder etwas arbeiten, doch klagte er im Oktober noch über stechende Schmerzen bei längerem Gehen und beim raschen Beugen des linken Knies.

Die von Herrn Prof. Haegler vorgenommene Untersuchung ergab: beim Stehen mit geschlossenen Beinen wird das linke Kniegelenk in ganz leichter Beugestellung gehalten, während das rechte Knie durchgedrückt wird. Beidseits besteht ein Genu valgum; links beträgt dessen Knickungswinkel $17^{\circ}$. Wird aber im Liegen das Knie passiv vollkommen gestreckt, so beträgt der Winkel dieser Abknickung nur $7-10^{\circ}$. Die aktive Streckung im linken Kniegelenk weist eine Insuffizienz von ca. $12^{0}$ auf, die aktive 
Beugung ist nicht wesentlich eingeschränkt. Passiv ist eine nahezu vollständige Streckung aber nur unter Anwendung von groBer Gewalt zu erreichen. Es können ferner bei

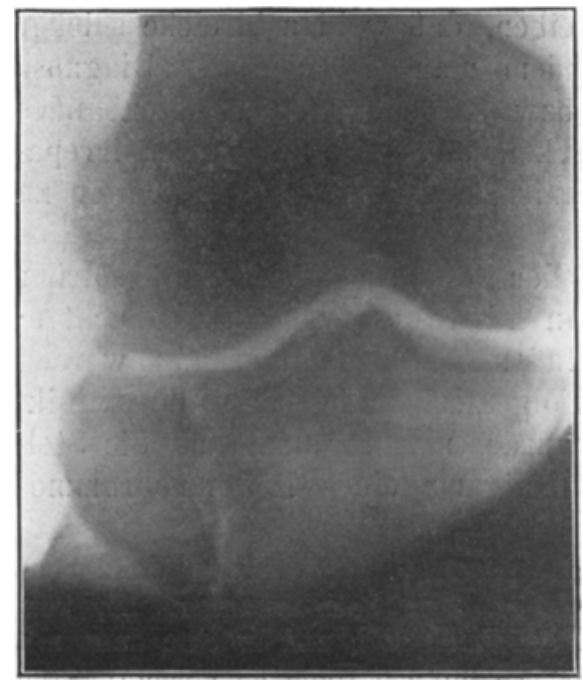

Fall 1.

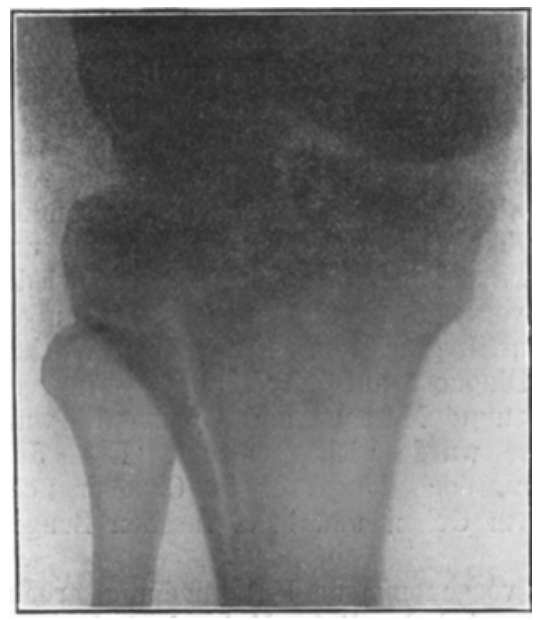

f'all 2. gestreckt gehaltenem Kniegelenk passiv leichte seitliche Bewegungen ausgeführt werden, die sclimerzlos sind, während derer aber ein Knarren im Knie wahrgenommen werden kann. - Die Außenseite des oberen Schien beinendes scheint etwas verdickt $\mathrm{zu}$ sein; sonst ist am linken Knie nichts Abnormes wahrnehmbar. Die Muskulatur des linken Beines zeigt eine ganz geringgradige Abmagerung. Der Gang ist unelastisch, hinkend. Die Röntgenuntersuchung ergibt, daß der Condylus externus tibiae abgebrochen und mit einer leichten Verschiebung nach abwärts angeheilt ist, In Anbetracht der abnormen Stellung des Beines und der vorhandenen Erschlaffung des Gelenkes wurde dem Patienten eine Einbuße der Erwerbsfähigkeit von 25 Proz. für 4 Monate, dann dauernd 15 Proz. zugesprochen.

II. 28 jäbriger Italiener. Er' war am 3. XI. 1908 von einem zusammenfallenden Gerüst $3 \mathrm{~m}$ hoch heruntergesprungen, Er ficl direkt auf die FüBe, kippte dann noch nach vorn um, wobei er das linke Knie auf einen Steinhaufen aufschlug. - Die am gleichen Tage von uns vorgenommene Untersuchung ergab beidseits ein Genu valgum; rechts beträgt der Knickungswinkel $9^{0}$, links $14^{0}$. Das linke Kniegelenk ist stark geschwollen; namentlich ist der obere Recessus prall gespannt; die Patella "tanzt"; Hautscharfungen fehlen. Die Gegend des Condylus externus tibiae ist sehr druckempfindlich; Krepitation und alonorme Beweglichkeit des Knochens sind nicht zu konstatieren. Bei gestrecktem Unterschenkel lassen sich beidseits abnorme seitliche passive Bewegungen in geringem Grade aus- 
führen, links deutlicher als rechts. Patient kann das Bein gestreckt in die Höhe halten; Stoß gegen die Fußsohle ist nicht schmerzhaift. Die Punktion des Gelenkes ergibt ca. $100 \mathrm{ccm}$ dünnflüssigen Blutes, auf welchem massenhaft Fettaugen schwimmen, - Nach der Punktion ist die aktive und passive Beweglichkeit im linken Kniegelenk etwas schmerzhaft, aber nicht erheblich eingeschränkt. Die Diagnose wurde auf Zerreißung des äußeren Seitenbandes gestellt, und wegen des in der Punktionsflüssigkeit vorhandenen Fettes die Wahrscheinlichkeit einer Gelenkfraktur der Tibia erwähnt.

Die Röntgenuntersuchung ergab eine Fraktur des Condylus externus tibiae; die Bruchlinie verläuft von der Mitte der Gelenkfläche direkt nach abwärts, wo sie ca. 4 Finger breit unterhalb der Gelenkfläche die Peripherie des Knochens auf der Außenseite erreicht. Der abgesprengte Condylus steht nur sehr wenig tiefer als normal.

Die Behandlung bestand in einem Kompressionsverband und Fixation des Beines auf einer Schiene. Nach 14 Tagen wurde dieser Verband entfernt; aktive und passive Bewegungen waren im Bein ohne Schmerzen ausfülırbar. /ur Korrektion der Valgusstellung wurde noch ein redressierender Gipsverband angelegt, mit welchem Patient 3 Wochen nach der Verletzung umherging. Mitte Dezember wurde der Gipsverband entfernt und Patient mit Massage und aktiven Bewegungen behandelt. Eine Ende Dezember vorgenommene Röntgenuntersuchung ergab, daß sich die Gelenkfläche des

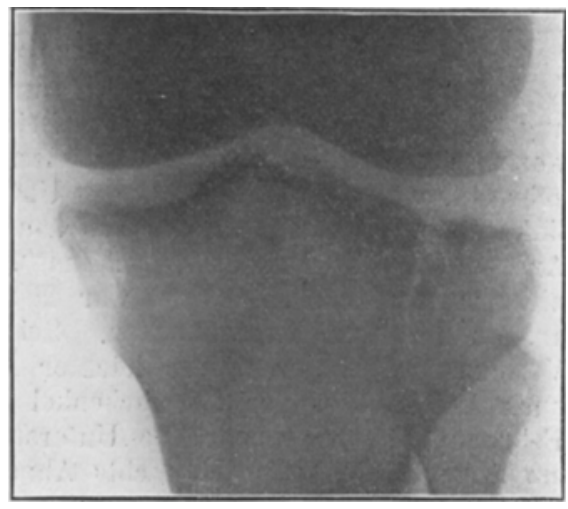

Fall 3. Condylus externus auf gleicher Höhe wie diejenige des internus befindet, daß also eine Heilung ohne Verschiebung des Fragmentes eingetreten ist.

III. 39 jälıriger Maschinenmeister, mit beidseitigem Genu valgum mittleren Grades, sprang im August 1908 von einer Lokomotive herunter, als diese auf einem stark ansteigenden Gerüst rückwärts rutschte. Wie er am Boden ankam, weiß er nicht mehr, doch muß er mit groBer Gewalt auf die Füße gefallen sein, denn man fand im Bahndamm zwei tiefe Eindrücke seiner Schuhe. Er lag dann wegen einer Fraktur des 1. Lendenwirbels and einer Fraktur des Os navieulare der rechten Hand und des linken Fußes 7 Wochen in einem Kantonsspital. Nach seiner Aussage war das rechte Knie geschwollen; die Wirbelsäule und der linke FuB wurden geröntget, das rechte Knie aber nicht, da dessen Beschwerden wegen der anderen schweren Verletzungen vollständig zurücktraten. 14 Wochen nach dem Unfall kam er hier zur Beobachtung; am Knie konnte außer einem Genu valgum nichts Abnormes konstatiert werden, doch klagte Patient immer noch über Schwächegefühl und Schmerzen im rechten Knie. 
Die Röntgenaufnahme ergibt wiederum eine Fraktur des äußeren Tibiacondylus, die ohne Verschiebung des Fragmentes (Bettruhe wegen der Wirbelfraktur!) geheilt ist.

Es handelt sich also in allen 3 Fällen um eine typische Verletzung, um eine Absprengung des Condylus externus tibiae, entstanden durch Fall auf die Füße, also durch indirekte Gewalt. Da bei allen drei Patienten ein doppelseitiges Genu valgum bestand, wird wohl die Entstehung dieser indirekten Fraktur mit den statischen Verhältnissen des Genu valgum zusammenhängen; doch davon später.

Bei einem 4. und 5. mir zur Verfügung stehenden Fall von Längsfraktur des Condylus externus tibiae war kein Genu valgum vorhanden; die Fraktur ist aber in Gegensatz zu den drei obigen Fällen durch direkte Gewalt entstanden.

IV. 28 jähriger Patient, wurde am 29. VII. 1908 von einem Automobil angefahren, welches ihn ans linke Bein traf. Patient wurde zu Boden geworfen und war kurze Zeit bewuBtlos. Er wurde dann zum nächsten Arzt gebracht, wo er einige Schritte mit Unterstützung gehen konnte. Das ganze linke Bein, speziell aber das Knie, sei stark geschwollen gewesen, Der Arzt sagte, das Bein sei „gelähmt". Eine Röntgenuntersuchung wurde nicht vorgenommen. Patient war fast 3 Monate zu Bett und fing danı an, an Krücken herumzugehen. Seit Neujahr (also 5 Monate nach der Verletzung) geht er wieder leichter Arbeit nach, ermüdet jedoch rasch; auch knickt ihm der Unterschenkel bisweilen nach außen um. Die im Februar 1909 vorgenommene Untersuchung ergibt: Beide Beine gerade; am linken Knie äußerlich nichts Abnormes sichtbar; leichte Atrophie der Muskulatur des linken Beines. Streckung und Beugung im linken Kniegelenk alstiv und passiv vollkommen ausführbar. Bei gestrecktem Knie kann man links leicht ausgiebige seitliche Bewegungen ausfuhhren, rechts nicht. - Die auf unsere Veranlassung vorgenommene Röntgenuntersuchung ergab eine Absprengung des äußeren Tibiacondyls, die, vermutlich wegen der lange andauernden Bettruhe, ohne wesentliche Dislokation geheilt ist. Wegen der ziemlich erheblichen Erschlaffung der Seitenbänder muB Patient einen Fixationsapparat tragen, in welchem er ohne Beschwerden herumgeht; sollte nicht bald Besserung eintreten, so wird die Verkürzung der Seitenbänder gemacht werden.

In einem 5. mir von Herrn Prof. Wilms zur Verfügung gestellten Fall war die.Fraktur dadurch entstanden, daß dem Patienten ein schweres Eisenblech gegen das linke Knie gefallen war und das Bein im Sinne eines Genu valgum abgeknickt hatte. Dadurch war der Condylus externus tibiae abgebrochen und stark nach unten und außen disloziert worden; außerdem war die Fibula im oberen Drittel frakturiert. Wegen der. starken Dislokation wurde das Fragment operativ reponiert und durch zwei Nägel an seinem Platz fixiert. Nach 3 wöchentlicher Bettruhe konnte Patient wieder umhergehen; er trug bloß noch eine Flanellbinde und wurde mit Massage und Gymnastil nachbehandelt. Die 8 Monate nach dem Unfall vorgenom- 
mene Nachuntersuchung ergab: links besteht ein Genu valgum mit einem Knickungswinkel von ca. $14^{0}$; das rechte Bein ist gerade. Das linke Kniegelenk ist nicht geschwollen, Krepitation ist in beiden Kniegelenken gleich stark fühlbar. Links kann man leicht ziemlich ausgiebige seitliche Bewegungen im Kniegelenk nachweisen. Die alstive Beugung ist größer als im rechten; die aktive Streckung ist nicht ganz bis zu $180^{\circ}$ ausführbar. Patient hat immer noch Schmerzen an der Bruchstelle (obschon er in keiner Unfallversicherung ist); er gibt an, daß die X-Beinstellung in letzter Zeit zugenommen habe. $\mathrm{Er}$ arbeitet den ganzen Tag als Schlossermeister, doch ermüdet er rasch bei längerer Arbeit am Ambos. - Herrn Prof. Wilms spreche ich meinen verbindlichsten Dank für die Erlauhnis zur Mitteilung dieses Falles aus.

Ich habe schon oben erwähnt, daß diese Frakturen nicht leicht zu diagnostizieren sind: der Umstand, daß von unseren 5 Fällen 3 primär nicht erkannt worden waren, bestätigt diese Ansicht.

Von den Symptomen ist der Gelenkerguß wohl immer vorhanden; in unserem ersten Fall, den wir zwar nicht frisch beobachten konnten, wird eine Gelenkschwellung vom zuerst behandelnden Arzt allerdings in Abrede gestellt. -- Konstant ist auch die Druckempfindlichkeit des äußeren Tibiaknorrens bei direktem Druck; Stoß gegen die Fußsohle verursachte in

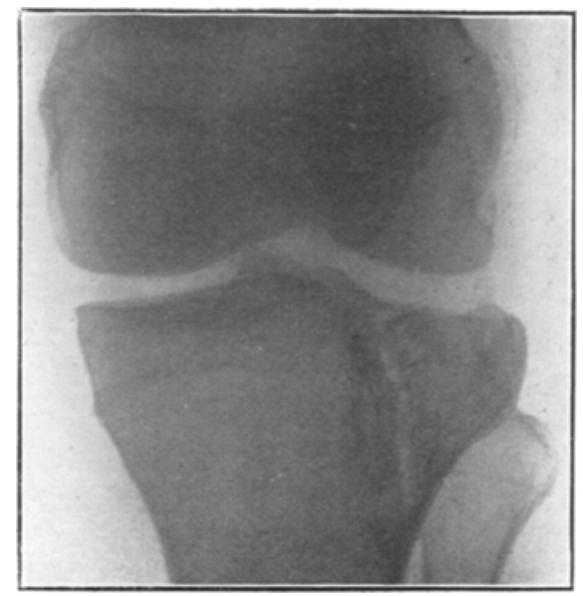

Fall 4.

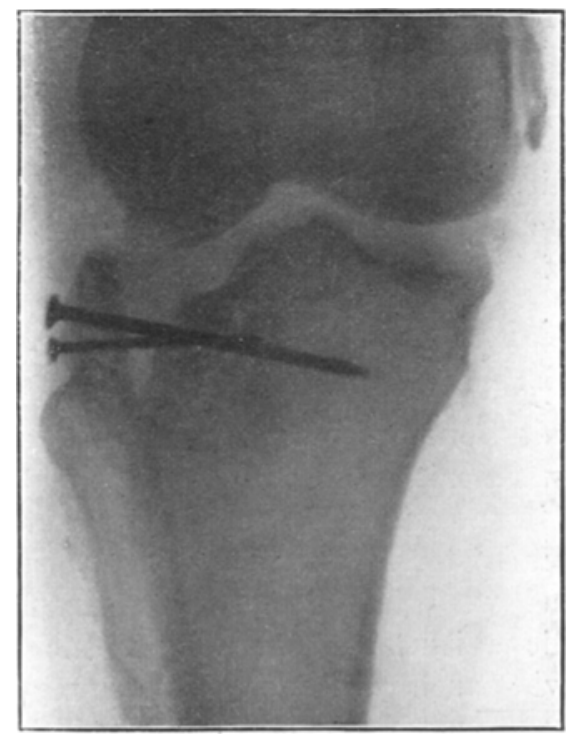

Fall 5. unserem zweiten Fall keine Schmerzen. - Abnorme Beweglichkeit des Fragmentes haben wir in unserem zweiten Fall nicht wahrnelimen 
können; die (Gestalt des Condylusfragmentes ist aber auch eine solche, daß die Schwierigkeit des Nach weises seiner Beweglichkeit erklärlich ist. - Krepitation ist wohl am besten bei seitlichen Bewegungen fühlbar; wir haben sie in unserem zweiten Fall nicht konstatiert. Lüw dagegen konnte sie in beiden Fällen nach weisen. Die aktive Beweglichkeit im Kniegelenk ist in allen Fällen sowohl durch die Schwellung als auch durch die Schmerzen etwas eingeschränkt. Die passive Beweglichkeit im Kniegelenk ist nur wenig eingeschränkt; Beugung und Streckung im Liegen wurde in unserem zweiten Fall ohne Beschwerden ausgeführt. Eine abnorme seitliche Beweglichkeit des Unterschenkels im Kniegelenk haben wir in allen Fällen feststellen können; sie ist - wie wir aus unseren Fällen ersehen haben - bei direkter Fraktur größer als bei indirekter. Die Erklärung hierfür ist leicht: bei direktem Bruch muß die Gewalt zuerst das innere Seitenband zerreißen, ehe der Femurcondyl den Tibiaknorren abdrücken kann; bei indirekter Fraktur (in welchen Fällen in der Regel ein Genu valgum vorhanden ist) kann infolge der Richtung der einwirkenden Gewalt von oben nach unten das Seitenband verschont bleiben. -- Bemerkenswert ist, daß alle Patienten trotz ihrer frischen Fraktur noch einige Schritte gehen konnten.

Angesichts der Vielartigkeit der Kniegelenksverletzungen bei ziemlich gleichartigen äußeren Frscheinungen leuchtet es ein, daß es nicht leicht ist. ans den erwähnten Symptowen die Diagnose dieser Fraktur zu stellen. Eine Röntgenaufnahme verschafft natürlich rasch Aufklürung. Ist aber eine solche aus äußeren Gründen nicht zn bewerkstelligen, so muß das Hanptgewicht anf die Art der Entstehung, auf die Druckempfindlichkeit des lateralen (ondylus, auf die abnorme seitliche Beweglichkeit und auf den Gelenkerguß gelegt werden; ergibt die Punktion des Gelenkes außerdem mit Fetttropfen untermischtes Blut, so ist auch dadurch die Diagnose mit größter Wahrscheinlichkeit zu stellen. -- Differentialdiagnostisch kommt bei der indirekten Entstehung in Betracht die Distorsio genu mit ev. Meniscus- oder Bänderverletzung; hierbei wird jedoch der Gelenkerguß nicht so groß sein und auch die seitliche Beweglichkeit nicht so stark hervortreten. Hier, wie auch bei der Entstehung durch direkte Gewalt - in welchem Fall die Möglichkeit einer bloßen Kontusion berücksichtigt werden muß - fehlt die exquisite Druckempfindlichkeit und der Bruchschmerz. - Das Vorhandensein eines Genu valgum soll uns an die Möglichkeit einer (ondylusfraktur erinnern. Bei den Kompressionsfrakturen der Tibia ist die Gelenkfläche ver- 
breitert; aber: Sicherheit in der Diagnose gibt uns nur das Röntgenogramm.

Die Therapie hat hauptsächlich die Verschiebung des Tibiafragmentes zu verhindern oder bei vorhandener Dislokation seine Reposition zu bewirken, damit nicht durch die Unebenheit der Gelenkfläche eine beständige Reibung und durch die Vermehrung der $\mathrm{X}$-Beinstellung eine Lockerung des Bandapparates stattfindet. Bei größerem Erguß empfiehlt sich die Punktion des Gelenkes, um eine Überdehnung der Kapsel zu verhüten. Das Blut ist in den ersten 3 Tagen noch größtenteils flüssig, kann also leicht entfernt werden. Nach der Punktion wird das Gelenk durch einen leichten Kompressionsverband vor einem erneuten Erguß geschützt und das ganze Bein unter Korrektion der Valgusstellung eingegipst. Nach ca. 4 Wochen wird der Verband entfernt, das Gelenk durch Heißluft und Massage, aktive und passive Bewegungen mobilisiert und auch der Atrophie der Oberschenkelmuskulatur entsprechende Aufmerksamkeit geschenkt. - Vor zu frühem Umhergehen muई entschieden gewarnt werden; die genaue Adaptation der Fragmente kann nur bei Bettruhe erhalten bleiben. Wenn unser erster Fall schon am 4. Tage ohne jeglichen Verband umherging, so ist das jedenfalls nicht ohne ungünstigen Einfluß auf die bleibende Stellung der Fragmente gewesen. Erhält man den Patienten frisch zur Behandlung, so ist in Fällen, in denen eime Dislokation des Fragmentes nach unten eingetreten ist, die Hebung dieses Knochenstückes mit darauffolgender Annagelung zur Verhütung stärkerer Valgusstellung zweifellos zu empfehlen. - Bei schlechtgeheilten Fällen mit Schlottergelenk soll man die Verkürzung der Seitenbänder vornehmen; anch ist es ev. angezeigt, eine kleine Knochenlamelle samt dem erschlafften Band abzumeißeln und höher oben anzunageln. Genügt dies alles nicht, so muß eben eine Schutzhülse getragen werden.

Die Prognose der Fraktur quoad restitutionem completam hängt in erster Linie von der Erkennung und richtigen Behandlung $a b$; aber auch bei entsprechendem Vorgehen ist sie nicht absolut günstig, handelt es sich doch um eine Gelenkfraktur, an welche sich ab und zu eine Arthritis deformans anschließt. Die funktionelle Restitution ist überhaupt eine auffallend langsame. Es rührt dies daher, daß die Fraktur größtenteils in der Epiphyse verläuft, und es sind für die ungünstige Heilung wohl auch folgende Ursachen in Betracht zu ziehen: 1. bildet der Knorpel keinen Callus; 2. reicht nach den Untersuchungen Bidders ${ }^{1}$ ) die osteogene Schicht des Peri-

1) Arch. f. klin. Chir, Bd. 22, S. 154. 
osts am oberen Tibiaende nur bis zur Grenze der Diaphyse. Nur die aus fibrillärem Bindegewebe bestehende äußere Schicht des Periosts setzt sich auf die Epiphyse bis zum Rande der Gelenkfläche fort und ist mit dem Epiphysenknorpel und Knochen fest verschmolzen. Es besitzt also die Epiphyse gar kein wahres zur Knochenprodnktion geeignetes Periost, ein Umstand, mit dem die lange andauernde Neigung dieser Frakturen zur Bildung eines Genu valgum direkt zusammenhängt. - Bei dauernder Verschiebung der Fragmente führt die Unebenheit der Gelenkfläche zu beständigen Reizungen im Gelenk, woraus sich schließlich eine chronische Arthritis entwickeln kann. Dazu kommt das Genu valgum, das durch die beständige Zerrung am Seitenband zu Schlottergelenk führt. Wie unser Fall IV zeigt, kann aber auch eine Schlaff heit des inneren Seitenbandes bei Befestigung in normaler Stellung weiterbestehen. Dies scheint jedoch, soviel wir einstweilen konstatieren konnten, nur bei direkten Verletzungen vorzukommen, bei welchen das Seitenband wohl schwerer lädiert wird, als bei der indirekten Entstehung.

Was den Entstehungsmechanismus betrifft, so war in den meisten in der Literatur niedergelegten Fällen die Fraktur durch indirekte Gewalteinwirkung entstanden; als Ursache findet man am häufigsten Fall aus der Höhe angegeben. Seltener kommen direkte Gewalten, wie Hufschlag oder Anschlagen des Knies, in Betracht. Der innere Condylus wird von der Verletzung häufiger betroffen als der äußere (15:10), wie auch der Condylus internus viel häufiger Kompressionsfrakturen erleidet als der äußere (16:4).

Mechanisch ist es leicht erklärlich, daß bei einem Fall auf die Füße der Condylus internus femoris, welcher der Schwerlinie des Körpers am nächsten liegt, auf den Condylus internus tibiae drückt, während der äußere erst bei stärkerem Anprall zur Wirkung kommt. Beim Genu valgum dagegen ist die äußere Seite stärker belastet als die innere. Bei einem Fall auf die Füße wird also der stärker belastete äußere Femurcondylus auf den Tibiacondylus drücken und ihn ev. zum Brechen bringen. Auch $\mathrm{Hoffa}^{2}$ ) ist der Ansicht, daß in den Fällen, in welchen der Condylus externus verletzt ist, vielleicht eine vorherige Knickung des Kniegelenks stattgefunden hat. Da in drei von unseren Fällen von indirekter Fraktur des äußeren Condylus ein doppelseitiges Genu valgum bestand, scheint es, da 13 die indirekte Fraktur des Condylus externus tibiae eine typischeVerletzung bei bestehendem Genuvalgum darstellt.

1) Orthop. Chir., S. 18. 
Auch für die Kompressionsfrakturen des äußeren Tibiacondyls, die Wagner bei Bergleuten fand, die stehend auf einer Förderschale in die Tiefe gestürzt waren, wird eine vorherige Abknickung im Sinne des Genu valgum angenommen. Die Patienten von Oenike') und Gurau ${ }^{2}$ ), die durch Fall auf die Füße Kompressionsfrakturen des äußeren Tibiacondyls erlitten hatten, waren mit doppelseitigem Genı valgum behaftet. Ob eine Längsfraktur oder ob ein Kompressionsbruch der Tibiacondylen zustande kommt, hängt wohl mehr von der Knochenbeschaffenheit des betreffenden Individuums ab, als von der einwirkenden Gewalt.

Wesentlich verschieden von dieser Ansicht stellt sich Anzoletti ${ }^{3}$ ) das Zustandekommen der indirekten Frakturen des Condylus externus tibiae vor. Er sagt: Wenn beim Stehen der Körper auf einem Bein ruht, so wird der Schwerpunkt von der Mittellinie auf den entsprechenden Fuß verlegt; dabei wird das belastete Bein in Adduktionsstellung zum Becken gebracht und diese durch eine Pronation des Fußes ausgeglichen. Je mehr die Adduktion und der Zug der ileotibialen Partie der Fascia lata vergrößert werden, um so stärker ist auch die Pronation des Fußes und um so dentlicher anch der Druck auf die Fibula. Wenn also jemand, während das eine Bein unbelastet ist, auf den andern Calcaneus auffällt, so wirken der Zug des Ligamentes und der Druck der Fibula in gleicher Zeit und in demselben Sinne auf den Condylus externus tibiae.

Die Möglichkeit der Entstehung dieser Fraktur durch Zug und Druck ist nicht ganz von der Hand zu weisen; doch scheint mir der ersterwähnte Entstehungsmodus viel einfacher zu sein; er stimmt auch mit den statischen Verhältnissen, speziell beim Genu valgum, vollständig überein. Anzolettis Fälle sind im Referat nicht näher beschrieben; die Originalarbeit war mir leider nicht zugänglich.

Daß diese Frakturen nicht so selten sind, wie überall angenommen wird, beweist der Umstand, daß wir im Laufe von 6 Monaten an unserem Material, das an Zahl demjenigen großer Krankenhäuser begreiflicherweise nachsteht, 4 solcher Fälle beobachten konnten. Es ist also nötig, an diese Fraktur zu denken und bei allen Knieverletzungen einen noch ausgiebigeren Gebrauch vom Röntgenverfahren zu machen als dies bisher geschehen ist.

1) 1)issert. Greifswald $18 \& 8$.

2) Deutsche Zeitschr. f. Chir. 1895, Bd. 41.

3) Arch. int. de chir. 1906, Bd. 3, Nr. 3, S. 267; ref. Schmidts Jahrbücher 1907, Bd. 295. 